\title{
The Lyapunov dimension of a nowhere differentiable attracting torus
}

\author{
JAMES L. KAPLAN, JOHN MALLET-PARET AND \\ JAMES A. YORKE
}

Department of Mathematics, Boston University, Boston, Massachusetts 02215, USA; Department of Mathematics, Michigan State University, East Lansing, Michigan 48824 and Lefschetz Centre for Dynamical Systems, Division of Applied Mathematics, Brown University, Providence, Rhode Island 02912, USA; Department of Mathematics and Institute for Physical Science and Technology, University of Maryland, College Park, Maryland 20742, USA

(Received 7 February 1983 and revised 10 September 1983)

Abstract. The fractal dimension of an attracting torus $T^{k}$ in $\mathbb{R} \times T^{k}$ is shown to be almost always equal to the Lyapunov dimension as predicted by a previous conjecture. The cases studied here can have several Lyapunov numbers greater than 1 and several less than 1

\section{Introduction}

This paper considers a pair of related problems. In $\$ 2$ we study the question of determining the dimension of the graph of the scalar function, defined by the series

$$
f(t)=\sum_{n=0}^{\infty} \lambda^{n} q\left(\beta^{n} t\right)
$$

where $0<\lambda<1$ and $\beta>1 / \lambda$ and $q$ is a smooth, non-constant, periodic or almost periodic, function. Our analysis and results are reminiscent of some classical work of Hardy on properties of the continuous, but nowhere differentiable, Weierstrass function [8]. Hardy studied various properties of (1.1) for $\lambda \beta \geq 1$ and $q(t)=\sin t$ or $q(t)=\cos t$. For Hausdorff dimension results see [2], [3], [5], [10], [12].

In $\S 3$ we turn our attention to the study of the 'dimension' of the strange attractor for the dynamical system defined by particular maps on the space $T^{2} \times \mathbb{R}$ where $T^{2}$ is the torus or more generally on $T^{k} \times \mathbb{R}$. The value derived is consistent with a conjecture stated by Frederickson et al. [7]. A preliminary version of their conjecture appeared in [9]. A discussion of the various meanings of dimension appears in [6].

Roughly speaking, the dimension of a set indicates the amount of information necessary to specify a given location with a desired precision. For a space $S$, let $N(\varepsilon)$ be the minimum number of points that can be chosen so that the $\varepsilon$ balls centred at these points cover the space. The fractal dimension (or capacity) of $S$ tells how $N(\varepsilon)$ grows as $\varepsilon$ shrinks to 0 . If the set is $d$-dimensional we expect 
$N(\varepsilon) \approx c e^{-d}$ for some constant $c$. Define the fractal dimension (i.e. capacity)

$$
\operatorname{dim}(S)=\lim _{\varepsilon \rightarrow 0^{+}} \frac{\log N(\varepsilon)}{\log 1 / \varepsilon} .
$$

whenever the limit exists.

Our principal result for $f$ defined in (1.1) is the following.

Theorem A. Assume

$$
q(t)=\sum_{i=1}^{N} q_{i} \cos \left(a_{i} t+\theta_{i}\right),
$$

for some real numbers $q_{i}, a_{i}, \theta_{i}$ where $i=1, \ldots, N$ for some $N$. Then either

(i) $\operatorname{dim}(\operatorname{graph} f)=2-|\log \lambda / \log \beta|$, or

(ii) $f$ is $C^{1}$.

Of course when $f$ is $C^{1}, \operatorname{dim}(\operatorname{graph} f)=1$. Case (i) must hold when $N=1$.

Our proof of this theorem deals with certain more general $q(t)$ and the proof characterizes when (ii) occurs in terms of a certain formal Fourier series. Our techniques are aimed at a rather different situation described in the theorems below and we cannot restrict attention to just those $q$ that have a Fourier series with a finite number of terms.

The only exceptional cases we know of where (ii) is satisfied are where there are constants $\beta_{0}$ and $\lambda_{0}$ and there is a $C^{1}$ function $r$ for which

$$
q(t)=\lambda_{0} r\left(\beta_{0} t\right)-r(t) \text {. }
$$

Then (ii) holds for $\lambda=\lambda_{0}$ and $\beta=\beta_{0}$. Roughly speaking for a given $\beta$ and $q$, (i) holds for all but a discrete set of $\lambda$.

In $\S 3$ we study the mapping $F: T^{k} \times \mathbb{R} \rightarrow T^{k} \times \mathbb{R}$ defined as follows. Let $T^{k}$ denote the $k$-dimensional torus and let $x$ be in $T^{k}$ where the coordinates of $x$ are all taken $\bmod 1$. Let $A$ denote a $k \times k$ matrix with integer coefficients satisfying $\operatorname{det} A=1$. Then $A$ can be considered as a mapping on $T^{k}$ when $A x$ is taken mod 1 in each coordinate, and this mapping is continuous on $T^{k}$. Let $y$ be real. Define $F: T^{k} \times \mathbb{R} \rightarrow$ $T^{k} \times \mathbb{R}$ by

$$
F(x, y)=(A x, \lambda y+p(x)),
$$

where $0<\lambda<1$ and $p: T^{k} \rightarrow \mathbb{R}$ is a smooth function of period 1 in each coordinate. The mapping $F$ determines the system

$$
\left(x_{n+1}, y_{n+1}\right)=F\left(x_{n}, y_{n}\right)
$$

for which the compact set $B=\left\{(x, y): x \in T^{k}\right.$ and $|y| \leq(1-\lambda)^{-1}$ sup $\left.|p|\right\}$ is mapped into itself by $F$. The attracting limit set for (1.4) is

$$
S=\bigcap_{i=1}^{\infty} F^{i}(B) \text {. }
$$

The form of $F$ in (1.4) implies that the attractor $S$ is of a special type. For each $x \in T^{k}$ there is exactly one $y \in \mathbb{R}$ such that $(x, y) \in S$ and $y=y(x)$ has an explicit formula

$$
y(x)=\sum_{n=1}^{\infty} \lambda^{n-1} p\left(A^{-n} x\right) .
$$


(Notice $A^{-1} x$ is well defined on $T^{k}$ since $\operatorname{det} A=1$.) Thus the graph of $y$, namely $\left\{(x, y(x)): x \in T^{k}\right\}$ is the attractor. To see the graph is invariant under $F$, compute

$$
\begin{aligned}
F(x, y(x)) & =(A x, p(x)+\lambda y(x)) \\
& =\left(A x, p\left(A^{-1} A x\right)+\sum_{n=1}^{\infty} \lambda^{n} p\left(A^{-n-1} A x\right)\right) \\
& =\left(A x, \sum_{n=1}^{\infty} \lambda^{n-1} p\left(A^{-n} A x\right)\right)=(A x, y(A x)) .
\end{aligned}
$$

Note also that each line $\{x\} \times \mathbb{R}$ is mapped to the line $\{A x\} \times \mathbb{R}$ and the map contracts distances on each line by a factor of $\lambda$. If we define $\phi: T^{k} \rightarrow \mathbb{R}$ by

$$
\phi(x)=\sum_{n=0}^{\infty} \lambda^{n} p\left(A^{-n} x\right)
$$

then $(x, y) \in S$ if and only if $(x, y)$ lies on the graph of $y=\phi\left(A^{-1} x\right)$. It is not hard to see that the dimension of the graph of $\phi$ is the same as that of $\phi \circ A^{-1}$. Thus our study of the dimension of the attractor $S$ is reduced to the study of the dimension of the graph of $\phi$. By restricting $\phi$ to appropriate 1-dimensional manifolds in $T^{k}$ we will be able to make use of the analysis of $\S 2$ to establish our main result, which we first state here for the $2 \times 2$ matrix $A_{*}$ where

$$
A_{*}=\left(\begin{array}{ll}
2 & 1 \\
1 & 1
\end{array}\right)
$$

The number $B_{1}=(3+\sqrt{5}) / 2$ plays a special role since $B_{1}$ and $B_{2}=1 / B_{1}$ (which is $(3-\sqrt{5}) / 2$ ) are eigenvalues of $A_{*}$.

TheOREM B. Let $A=A_{*}$. Let $p: T^{2} \rightarrow R$ be $C^{3}$ and assume $\lambda \in\left(B_{2}, 1\right)$. Then either

(i) $\phi$ is nowhere differentiable and

$$
\operatorname{dim}(\operatorname{graph} \phi)=3-\left|\frac{\log \lambda}{\log B_{2}}\right|,
$$

or

(ii) $\phi$ is smooth and $\operatorname{dim}(\operatorname{graph} \phi)=2$.

The right side of (1.8) is a number strictly between 2 and 3. For a given non-constant $p$ condition (i) occurs for nearly every choice of $\lambda$ (i.e. all but a discrete set of $\lambda$ ). For $\lambda$ fixed there is an infinite number of independent relationships that the Fourier series coefficients of $p$ must satisfy in order for (ii) to be satisfied. In fact if $w_{2}$ is an eigenvector for $B_{2}$ and we let $q(t)=p\left(t w_{2}\right)$, then

$$
\sum_{n=-\infty}^{\infty} \lambda^{n} q\left(B_{2}^{-n} t\right) \equiv 0
$$

if and only if (ii) is satisfied. (We show this sum is well defined.) The set of $p$ in $C^{3}$ satisfying (ii) is a closed subspace having an infinite dimensional complementary subspace. We are justified in saying that it is 'infinitely likely' the $p$ satisfies (i). There are no intermediate cases where the fractal dimension is greater than 2 but is less than the number in (1.8). When $0<\lambda<B_{2}$, conclusion (ii) always holds. 
When the domain of $y(x)$ is restricted to a line or line segment on the torus, the graph is easy to construct. For $p(x)=1+\cos (2 \pi u)$ where $x=(u, v)$ is restricted to the segment (or circle) $x=(u, 0.5), 0 \leq u \leq 1$, the resulting curve is essentially Weierstrass' nowhere differentiable curve, and Moser used this fact to show that the attractor was nowhere differentiable [13], when $p(x)=\cos (2 \pi u)$.

Theorem B corresponds to a part of conjecture 2 of [7] for the specific dynamical system (1.4), (1.5). In order to interpret this result and to motivate formula (1.8), we must introduce some additional concepts.

For any $k$ vectors $v_{1}, v_{2}, \ldots, v_{k}$ in $R^{n}, k \leq n$, let $\operatorname{vol}_{k}\left(v_{1}, \ldots, v_{k}\right)$ denote the $k$-dimensional volume of the $k$-dimensional parallelepiped that has a vertex at 0 and $v_{1}, \ldots, v_{k}$ as edges. Here $\operatorname{vol}_{1}(v)=\|v\|$. Write

$$
A_{m}=D\left(f^{m}(x)\right)
$$

for the $n \times n$ matrix of partial derivatives of the $m$ 'th iterate of $f: \mathbb{R}^{n} \rightarrow \mathbb{R}^{n}$. For $x \in \mathbb{R}^{n}$, let

$$
\delta_{k}\left(x, v_{1}, \ldots, v_{k}\right)=\lim _{m \rightarrow \infty}\left[\operatorname{vol}_{k}\left(A_{m} v_{1}, \ldots, A_{m} v_{k}\right)\right]^{1 / m}
$$

whenever this limit exists. If almost every choice of $v_{1}, v_{2}, \ldots v_{k}$ and almost every $x$ (with respect to Lebesgue measure) yields the same number then we say that the mapping $f$ has a $k$-dimensional growth rate and we denote this value by $\delta_{k}$. When these rates exist for $k=1,2, \ldots, n$, write

$$
\begin{aligned}
& L_{1}=\delta_{1} \\
& L_{i}=\delta_{i} / \delta_{i-1} \quad i=2, \ldots, n .
\end{aligned}
$$

We say $L_{i}$ is the $i$ 'th Lyapunov number of $f$. It can be seen that $L_{1} \geq L_{2} \geq \cdots \geq L_{n}$. If $f$ is linear the Lyapunov numbers exist and they are the absolute values of the eigenvalues of $f$. Next if $L_{1} \geq 1$ let

$$
\begin{aligned}
& \rho_{k}=k+\frac{\log \delta_{k}}{\left|\log L_{k+1}\right|} \quad k=1,2, \ldots, n-1, \\
& \rho_{n}=n .
\end{aligned}
$$

Define $m=\max \left\{i: \delta_{i} \geq 1\right\}$. If $L_{1}<1$, we define $\rho_{m}=0$. Notice that if $m<n$ then $m \leq \rho_{m}<m+1$. We call $\rho_{m}$ the Lyapunov dimension and write $\operatorname{dim}_{\text {Lyap }} S$. Notice that this concept of dimension depends upon the mapping $f$ as well as the set $S$.

It often happens that almost every point in a neighbourhood of an ergodic attractor will yield the same Lyapunov numbers. We will say that the Lyapunov numbers are absolute if every $x$ (with no exception in some neighbourhood of the attractor) yields the same Lyapunov numbers.

In [7] we provided several examples and some heuristic arguments which suggested this imprecisely stated conjecture:

Conjecture. For a 'typical' $f$ with $L_{1}>1$, the attractor satisfies

$$
\operatorname{dim} S \geq \operatorname{dim}_{\text {Lyap }} S \text {; }
$$

for a 'typical' $f$ with absolute Lyapunov numbers the attractor $S$ satisfies

$$
\operatorname{dim} S=\operatorname{dim}_{\text {Lyap }} S \text {. }
$$


When Lyapunov numbers exist but are not absolute a different definition of dimension must be substituted for the fractal dimension. See [6], [11] and [7]; [1] gives our most careful formulation of what 'typical' means.

The function $F$ in (1.4) has absolute Lyapunov numbers and they are the absolute values of the eigenvalues of $A$ and also $|\lambda|$. For $A=A_{*}$ they are $(3+\sqrt{5}) / 2, \lambda$ and $(3-\sqrt{5}) / 2$, and $(1.8)$ is simply the Lyapunov dimension of $S$. For $\lambda \leq B_{2}$, equation 1.10 holds since $\operatorname{dim} S=2$.

The methods used in the example in theorem $B$ extend easily to a larger class of examples. Let $A$ be a $k \times k$ matrix with integer coefficients and determinant $=1$. Assume $A$ has $k$ real eigenvalues $B_{i}$ with corresponding eigenvectors $w_{i}$ which we assume are linearly independent, and assume

$$
\left|B_{1}\right| \geq \cdots \geq\left|B_{k-1}\right|>\left|B_{k}\right| \text {. }
$$

Write $B_{*}$ for $\left|B_{k}\right|$ and let $w_{*}$ be the corresponding eigenvector of $A$.

THEOREM C. Let $p$ be a $C^{k+1}$ function. When $B_{*}<\lambda<1$, it is 'infinitely likely' that $\phi$ is nowhere differentiable and

$$
\operatorname{dim}(S)=\operatorname{dim}_{\text {Lyap }}(S)
$$

and when (1.10) is not true, (1.9) is true where $q(t)=p\left(t w_{*}\right)$, and $B_{2}$ is replaced by $B_{*}$.

As before, when $\lambda \leq\left|B_{*}\right|$, the attracting torus is smooth and both sides of (1.10) equal $k$.

To simplify notation, we present the arguments for theorem $B$, and leave to the reader the minor modifications needed for theorem $C$. We remark that we do not know how to extend our results to the case $p: T^{k} \rightarrow \mathbb{R}^{m}$ where $m>1$, though results in [7] certainly suggest it may still be valid.

$\$ 2$ concentrates on the Fourier series arguments needed for theorems A, B and C. It is relatively easy to show that the dimension of the graph of $f$ cannot be larger than what is given in (i) of theorem $\mathrm{A}$ (proposition 2.9). The primary problem is to show that if there is a Fourier coefficient of $q(t)$ that prevents $f$ from being smooth, then graph $(f)$ must have dimension at least $2-|\log \lambda / \log \beta|$. $^{\prime}$ Hardy had a similar problem in his analysis of Weierstrass' nowhere differentiable function, but he needed less uniformity and so his methods have been of no use to us, even in the simplest case where $q(t)=\cos t$. $\$ 3$ shows how theorems B and C reduce to theorem $\mathrm{A}$ with $\beta=B_{*}$, via a description of the geometry.

\section{Nowhere differentiable functions}

In this section we establish conditions under which $f$ is smooth and thus will have a one dimensional graph. We begin by considering an almost periodic non-constant function $q: \mathbb{R} \rightarrow \mathbb{R}$. We assume throughout this section that $q$ is $C^{1}$. Without loss of generality we may assume $q(0)=0$, replacing $q(x)$ by $q(x)-q(0)$ if necessary, causing a vertical translation of the graphs studied. Let $\lambda \in(0,1), \beta \in(1 / \lambda, \infty)$ be constants. Denote the Fourier series of $q$ by

$$
q(t) \sim \sum_{a} q_{a} e^{i a t}
$$


where

$$
q_{a}=\lim _{T \rightarrow \infty} \frac{1}{2 T} \int_{-T}^{+T} q(t) e^{-i a t} d t .
$$

From the theory of almost periodic functions $\left\{a: q_{a} \neq 0\right\}$ is countable and $q_{-a}=\bar{q}_{a}$; see [4]. The symbol $\sim$ may be read 'has the Fourier series'. This sum (2.1) is formal in that it does not necessarily converge.

Define the continuous function

$$
f(t)=\sum_{n=0}^{\infty} \lambda^{n} q\left(\beta^{n} t\right)
$$

Let $\alpha=\log (1 / \lambda) / \log \beta$. Notice the role of this $\alpha$ in theorem $\mathrm{A}(\mathrm{i})$. It will remain a regular participant. Observe that

$$
0<\alpha<1 \text { and } \lambda=\beta^{-\alpha} .
$$

Define the doubly infinite formal sum $g(t)$ by

$$
g(t)=\sum_{n=-\infty}^{\infty} \lambda^{n} q\left(\beta^{n} t\right)
$$

We interpret $g$ formally as having the Fourier series

$$
g(t) \sim \sum_{\sigma} g_{\sigma} e^{i \sigma t}
$$

where

$$
g_{\sigma}=\sum_{k=-\infty}^{\infty} \lambda^{k} q_{\sigma \beta^{-k}}
$$

Note that $q_{a}$ is defined for all $a \in \mathbb{R}$ even though it is 0 except for countably many $a$ 's. Here we use only those $a$ of the form $a=\sigma \beta^{-k}$. The coefficients $g_{\sigma}$ can be well defined if the sum (2.6) converges. That requires $q_{\sigma \beta^{-k}}$ to go to 0 sufficiently rapidly as $k \rightarrow-\infty$. Note again the coefficient $g_{\sigma}$ can be well defined without the series (2.5) being convergent. In order to guarantee convergence we assume the following hypothesis:

(H1) $\sum_{a}\left|q_{a}\right||a|^{\alpha}<\infty$.

The objective of the next few pages is to prove that if $(\mathrm{H} 1)$ is satisfied then

$$
g_{\sigma}=0 \text { for all } \sigma \Rightarrow f \text { is } C^{1} \text {. }
$$

The graph of $f$ must of course then have dimension equal to 1 . Condition (H1) clearly implies that the sum in $(2,6)$ is absolutely convergent since the subscripts being summed in (2.6) are $a=\sigma \beta^{-k}$, so

$$
|a|^{\alpha}=|\sigma|^{\alpha} \beta^{-k \alpha}=|\sigma|^{\alpha} \lambda^{k}
$$

since $\beta^{-\alpha}=\lambda$. Hence the sum in (H1) equals $\sum_{1 \leq|\sigma|<\beta} \sum_{k=-\infty}^{\infty}|\sigma|^{\alpha} \lambda^{k} q_{\sigma \beta^{-k}}$ since any $a \neq 0$ can be written uniquely as $a=\sigma \beta^{-k}$ with $1 \leq|\sigma|<\beta$. Moreover it follows that

(i) $g_{\beta \sigma}=\lambda g_{\sigma}$;

(ii) $g_{-\sigma}=\overline{\boldsymbol{g}}_{\sigma}$.

Again writing $a>0$ as $\sigma \beta^{-k}$ for some integer $k$ and some $\sigma \in[1, \beta$ ), we may express a sum over all $a>0$ as a double sum over $1 \leq \sigma<\beta$ and over all integers $k$. Further, 
$\left|\lambda^{k} q_{\sigma \beta^{-k}}\right|$ can then be written $\left|(a / \sigma)^{\alpha} q_{a}\right|$ which is less than or equal to $a^{\alpha}\left|q_{a}\right|$. Hence we have

(iii) $\sum_{1 \leq \sigma<\beta}\left|g_{\sigma}\right| \leq \sum_{a>0}\left|q_{a}\right| a^{\alpha}<\infty$.

Note that (H1) always holds if $q(t)$ is a finite $\operatorname{sum} \sum q_{a} e^{i a t}$, such as a trigonometric polynomial. Roughly speaking, the sum in (H1) will converge if $q$ is sufficiently smooth in some sense. Indeed, if $q$ is quasi-periodic with $N$ independent frequencies (i.e. $q(t)=p\left(\mu_{1} t, \ldots, \mu_{N} t\right)$ where $p: T^{N} \rightarrow \mathbb{R}$ is a function on the $N$-torus and $\mu_{1}, \ldots, \mu_{N}$ are linearly independent over the rationals) then (H1) will hold if $p$ is $C^{N+1}$. In $\S 3$ this is shown for $N=2$, and the proof there generalizes for arbitrary $N$. When $p$ is defined on $T^{k}$ as in theorem $\mathrm{C}$, the number of independent frequencies satisfies $N \leq k$, so for (H1) to hold, it suffices for $p$ to be in $C^{k+1}$.

Next we define the function

$$
h(t)=\sum_{n=-\infty}^{-1} \lambda^{n} q\left(\beta^{n} t\right)
$$

This function is continuous because its series converges uniformly on compact $t$-intervals. To show this recall $q(0)=0$ and note that if $K_{1}>0$, then since $q \in C^{1}$ there must exist $K_{2}$ such that $\left|q^{\prime}(t)\right| \leq K_{2}$, whenever $|t| \leq K_{1}$. This implies that $|q(t)| \leq K_{2} t$, and similarly

$$
\left|\lambda^{n} q\left(\beta^{n} t\right)\right| \leq K_{2}(\lambda \beta)^{n} t,
$$

so the series in (2.8) converges since $\lambda \beta>1$ and $n$ is negative. It follows now that $g(t)=f(t)+h(t)$ is a continuous function. Also observe that $g(\beta t)=\lambda^{-1} g(t)$. We must relate the properties of the continuous function $g(t)$ to the Fourier series in (2.5), and we are especially interested in the case where all $g_{\sigma}=0$.

LeMma 2.1. Assume (H1) holds. Then $h(t)$ is a $C^{1}$ function.

Proof. Define

$$
\eta(t)=\sum_{n=-\infty}^{-1}(\lambda \beta)^{n} q^{\prime}\left(\beta^{n} t\right)
$$

This is a continuous function, since the series converges uniformly. Let

$$
\eta_{k}(t)=\sum_{n=-k}^{-1}(\lambda \beta)^{n} q^{\prime}\left(\beta^{n} t\right) .
$$

Thus for any $t$,

$$
\int_{0}^{t} \eta_{k}(s) d s \rightarrow \int_{0}^{t} \eta(s) d s, \quad \text { as } k \rightarrow \infty .
$$

On the other hand

$$
\int_{0}^{t} \eta_{k}(s) d s=\sum_{n=-k}^{-1} \lambda^{n} q\left(\beta^{n} t\right) \rightarrow h(t),
$$

hence $h(t)=\int_{0}^{t} \eta(s) d s$ is $C^{1}$.

Proposition 2.2. Assume (H1) holds. Then the formal Fourier coefficients $g_{\sigma}$ are all 0 if and only if $g(t) \equiv 0$ as a continuous function. 
Proof. We begin by defining the auxiliary functions $\psi^{ \pm}(y)$ by

$$
g( \pm t)=t^{\alpha} \psi^{ \pm}(\log t), \quad t>0
$$

Because $g(\beta t)=\lambda^{-1} g(t)$ it follows that

$$
\psi^{ \pm}(y+\log \beta)=\psi^{ \pm}(y)
$$

for all $y \in \mathbb{R}$, and $\psi^{ \pm}$is continuous. The continuous function $g(t)$ is zero for all $t$ if and only if the periodic continuous functions $\psi^{ \pm}(y)$ are both zero for all $y \in \mathbb{R}$. This in turn is true if and only if each Fourier coefficient $\psi_{n}^{ \pm}=0$. Thus to prove proposition 2.2 we shall show $g_{\sigma}=0$ for all $\sigma$ if and only if $\psi_{n}^{+}=\psi_{n}^{-}=0$ for all $n$, where

$$
\psi^{ \pm}(y) \sim \sum_{n=-\infty}^{\infty} \psi_{n}^{ \pm} e^{2 \pi i n y / \log \beta}
$$

is the Fourier series for $\psi^{ \pm}$, that is,

$$
\psi_{n}^{ \pm}=\frac{1}{\log \beta} \int_{0}^{\log \beta} \psi^{ \pm}(y) e^{-2 \pi i n y / \log \beta} d y .
$$

We will need the following lemma.

LEMMA 2.3.

$$
\left.\begin{array}{l}
\psi_{n}^{+}=\sum_{a>0}\left(q_{a} \Omega_{n}+q_{-a} \bar{\Omega}_{-n}\right) a^{\alpha} e^{2 \pi i n \log a / \log \beta} \\
\psi_{n}^{-}=\sum_{a>0}\left(q_{a} \bar{\Omega}_{-n}+q_{-a} \Omega_{n}\right) a^{\alpha} e^{2 \pi i n \log a / \log \beta}
\end{array}\right\}
$$

where the number $\Omega_{n}$ depends on $\alpha$ and $\beta$ and is given by the formula

$$
\Omega_{n}(\alpha, \beta)=\frac{1}{\log \beta} \int_{0}^{\infty}\left(e^{i t}-1\right) t^{-\alpha-1-(2 \pi i n / \log \beta)} d t .
$$

The proof will be delayed until we have shown how this proves proposition 2.2.

We may write any $a>0$ uniquely as $a=\sigma \beta^{-k}$ where $1 \leq \sigma<\beta$. From (2.6) and (2.9) it follows in this notation that

$$
\begin{aligned}
\psi_{n}^{+} & =\sum_{1 \leq \sigma<\beta} \sum_{k=-\infty}^{\infty}\left(q_{a} \Omega_{n}+q_{-a} \overline{\Omega_{-n}}\right) a^{\alpha} e^{2 \pi i n \log a / \log \beta} \\
& =\sum_{1 \leq \sigma<\beta} \sum_{k=-\infty}^{\infty}\left(q_{\sigma \beta^{-k}} \Omega_{n}+q_{-\sigma \beta} \overline{\Omega_{-n}}\right) \lambda^{k} \sigma^{\alpha} e^{2 \pi i n \log \sigma / \log \beta} \\
& =\sum_{1 \leq \sigma<\beta}\left(g_{\sigma} \Omega_{n}+g_{-\sigma} \overline{\Omega_{-n}}\right) \sigma^{\alpha} e^{2 \pi i n \log \sigma / \log \beta},
\end{aligned}
$$

and similarly

$$
\psi_{n}^{-}=\sum_{1 \leq \sigma<\beta}\left(g_{\sigma} \overline{\Omega_{-n}}+g_{-\sigma} \Omega_{n}\right) \sigma^{\alpha} e^{2 \pi i n \log \sigma / \log \beta} .
$$

Thus if $g_{\sigma}=0$ for each $\sigma$, then $\psi_{n}^{+}=\psi_{n}^{-}=0$ for each $n$, which in turn implies that $g(t) \equiv 0$ as a continuous function. 
Before we establish the converse, we show that $\operatorname{Im}\left(\Omega_{n} \Omega_{-n}\right) \neq 0$; we do this by evaluating $\Omega_{n}$ explicitly in terms of the $\Gamma$-function. Integration by parts yields

$$
\begin{aligned}
\Omega_{n} & =\frac{1}{\log \beta} \int_{0}^{\infty}\left(e^{i t}-1\right) t^{-\alpha-1-(2 \pi i n / \log \beta)} d t \\
& =\frac{i}{(\log \beta)(\alpha+(2 \pi i n / \log \beta))} \int_{0}^{\infty} e^{i t} t^{-\alpha-(2 \pi i n / \log \beta)} d t .
\end{aligned}
$$

Shift the path of integration in the complex plane from the positive real axis $(0 \leq x<\infty)$ to the positive imaginary axis $\{y: y=i t, 0 \leq t<\infty\}$. In doing so, note that

$$
(\text { it })^{-\alpha-(2 \pi i n / \log \beta)}=e^{-(\pi i \alpha / 2)+\left(\pi^{2} n / \log \beta\right)} t^{-(\alpha-\pi i n / \log \beta)} \text {. }
$$

We now have

$$
\begin{aligned}
\Omega_{n} & =\frac{-e^{-(\pi i \alpha / 2)+\pi^{2} n / \log \beta}}{(\log \beta)(\alpha+(2 \pi i n / \log \beta))} \int_{0}^{\infty} e^{-t} t^{-\alpha-(2 \pi i n / \log \beta)} d t \\
& =\frac{e^{-(\pi i \alpha / 2)+\pi^{2} n / \log \beta}}{\log \beta} \Gamma\left(-\alpha-\frac{2 \pi i n}{\log \beta}\right),
\end{aligned}
$$

and so

$$
\Omega_{n} \Omega_{-n}=\frac{e^{-\pi i \alpha}}{(\log \beta)^{2}}\left|\Gamma\left(-\alpha-\frac{2 \pi i n}{\log \beta}\right)\right|^{2} .
$$

Now $1 /(\log \beta)^{2} \mid \Gamma\left(-\alpha-\left.(2 \pi \operatorname{in} / \log \beta)\right|^{2}\right.$ is real and non-zero since $1 / \Gamma(z)$ is an entire function. Thus, $\operatorname{Im}\left(\Omega_{n} \Omega_{-n}\right) \neq 0$, since $0<\alpha<1$.

Now assume $g(t) \equiv 0$ as a continuous function; then (as we will show later)

$$
\sum_{1 \leq \sigma<\beta} g_{\sigma} \sigma^{\alpha} e^{2 \pi i n \log \sigma / \log \beta}=0
$$

for each $n$. Introduce the variable $s=\log \sigma / \log \beta \in[0,1)$, where $\sigma \in[1, \beta)$, and define a measure $\mu$ on $[0,1)$ as follows: the measure $\mu$ consists of point masses of weight

$$
g_{\sigma} \sigma^{\alpha}=g_{\beta^{s}} \beta^{\alpha s}
$$

at the points $s \in[0,1)$. This defines a finite measure since $\sum_{1 \leq \sigma<\beta}\left|g_{\sigma} \sigma^{\alpha}\right|<\infty$. Moreover (2.10) becomes

$$
0=\int_{[0,1)} e^{2 \pi i n s} d \mu(s) \quad \text { for each } n .
$$

But this implies $\int_{[0,1)} F(s) d \mu(s)=0$ for every 1-periodic continuous function, which implies the measure $\mu$ must be the zero measure. Hence $g_{\sigma}=0$ if $\sigma \in[1, \beta)$; a similar argument involving the $g_{-\sigma}$ yields $g_{-\sigma}=0$ for all $\sigma \in[1, \beta)$.

Now (2.10) and the corresponding equation for $\boldsymbol{g}_{-\sigma}$ are obtained by taking suitable linear combinations of $\psi_{n}^{+}$and $\psi_{n}^{-}$, which are known to be zero since $g(t) \equiv 0$; to do this we need a certain $2 \times 2$ determinant to be non-zero. But this follows from the fact that $\Omega_{n} \Omega_{-n}$ is not real, for then

$$
\Omega_{n} \Omega_{n}-\bar{\Omega}_{-n} \bar{\Omega}_{-n}=\Omega_{-n}^{-2}\left(\left(\Omega_{n} \Omega_{-n}\right)^{2}-\left|\Omega_{-n}\right|^{4}\right)
$$


so $g_{\sigma}=g_{-\sigma}=0$ for each $\sigma \in[1, \beta)$. And from (2.7) it follows $g_{\sigma}=0$ for each $\sigma \in \mathbb{R}$. This proves proposition 2.2, provided we can establish lemma 2.3 .

Proof of lemma 2.3. We prove only the formula for $\psi_{n}^{+}$. From the definition of Fourier coefficient

$$
\begin{aligned}
\psi_{n}^{+} & =\frac{1}{\log \beta} \int_{0}^{\log \beta} \psi^{+}(y) e^{-2 \pi i n y / \log \beta} d y \\
& =\frac{1}{\log \beta} \int_{0}^{\log \beta} e^{-\alpha y} g\left(e^{y}\right) e^{-2 \pi i n y / \log \beta} d y \\
& =\frac{1}{\log \beta} \int_{1}^{\beta} g(t) t^{-\alpha-1-(2 \pi i n / \log \beta)} d t .
\end{aligned}
$$

Formal substitution of (2.5) and (2.6) would lead to the desired formula (2.9), if we did not have to worry about convergence. In general, set

$$
\begin{gathered}
q_{\varepsilon}(t)=\frac{1}{\varepsilon} \int_{1}^{t+\varepsilon} q(s) d s-\varepsilon \int_{t}^{t+(1 / \varepsilon)} q(s) d s . \\
g^{\varepsilon}(t)=\sum_{k=-\infty}^{\infty} \lambda^{k}\left[q_{\varepsilon}\left(\beta^{k} t\right)-q_{\varepsilon}(0)\right] \\
\psi_{n}^{\varepsilon}=\frac{1}{\log \beta} \int_{1}^{\beta} g^{\varepsilon}(t) t^{-\alpha-1-(2 \pi i n / \log \beta)} d t .
\end{gathered}
$$

Claim. $g^{\varepsilon}(t) \rightarrow g(t)$ pointwise, and $\sup \left|g^{\varepsilon}(t)\right|<\infty$ for $0<\varepsilon \leq 1,1 \leq t \leq \beta$.

We will proceed as if this claim is valid. We will establish it later in the proof. By the Lebesgue Dominated Convergence Theorem

$$
\psi_{n}^{\varepsilon} \rightarrow \psi_{n}^{+} \quad \text { as } \varepsilon \rightarrow 0 .
$$

Let us now calculate explicitly the formula for $\psi_{n}^{\varepsilon}$ in terms of the Fourier coefficients of $q(t)$. We have

$$
q_{\varepsilon}(t)-q_{\varepsilon}(0)=\sum_{a} q_{a} \gamma(a, \varepsilon)\left(e^{i a t}-1\right)
$$

which is an absolutely convergent series, by ( $\mathrm{Hl}$ ), and because

$$
\gamma(a, \varepsilon)=\frac{e^{i a \varepsilon}-1}{i a \varepsilon}-\frac{e^{i a / \varepsilon}-1}{i a / \varepsilon}
$$

is of order $O(a)$ as $a \rightarrow 0$, for any fixed $\varepsilon \rightarrow 0$ (though this bound is not uniform in $\varepsilon)$. By direct substitution this gives

$$
\begin{aligned}
\psi_{n}^{\varepsilon} & =\frac{1}{\log \beta} \sum_{k=-\beta}^{\infty} \int_{1}^{\beta} \lambda^{k}\left[q_{\varepsilon}\left(\beta^{k} s\right)-q_{\varepsilon}(0)\right] s^{-\alpha-1-(2 \pi i n / \log \beta)} d s \\
& =\frac{1}{\log \beta} \sum_{k=-\infty}^{\infty} \sum_{a} \lambda^{k} q_{a} \gamma(a, \varepsilon) \int_{1}^{\beta}\left(e^{i a \beta^{k}}-1\right) s^{-\alpha-1-(2 \pi i n / \log \beta)} d s \\
& =\frac{1}{\log \beta} \sum_{a} \sum_{k=-\infty}^{\infty} q_{a} \gamma(a, \varepsilon) \int_{\beta^{k}}^{\beta^{k+1}}\left(e^{i a s}-1\right) s^{-\alpha-1-(2 \pi i n / \log \beta)} d s,
\end{aligned}
$$

where we were able to interchange the limits of summation because the series is 
absolutely convergent. This, in turn, is equal to

$$
\begin{aligned}
& \sum_{a} q_{a} \gamma(a, \varepsilon) \frac{1}{\log \beta} \int_{0}^{\infty}\left(e^{i a s}-1\right) s^{-\alpha-1-(2 \pi i n / \log \beta)} d s \\
= & \sum_{a} q_{a} \gamma(a, \varepsilon) \frac{|a|^{\alpha+(2 \pi i n / \log \beta)}}{\log \beta} \int_{0}^{\infty}\left(e^{i s \operatorname{sgn}(a)}-1\right) s^{-\alpha-1-(2 \pi i n / \log \beta)} d s \\
= & \sum_{a>0} a^{\alpha} e^{2 \pi i n \log a / \log \beta}\left[q_{a} \Omega_{n} \gamma(a, e)+q_{-a} \bar{\Omega}_{-n} \gamma(-a, \varepsilon)\right] .
\end{aligned}
$$

Finally, we take the limit in (2.13) as $\varepsilon \rightarrow 0$. The left hand side $\psi_{n}^{\varepsilon} \rightarrow \psi_{n}^{+}$as noted. We have:

and consequently

$$
\begin{array}{cc}
\sum_{a}\left|q_{a}\right||a|^{\alpha}<\infty & \text { by (H1), } \\
\sup |\gamma(a, \varepsilon)|<\infty & \text { for } a, \varepsilon \in \mathbb{R} \\
\lim _{\varepsilon \rightarrow 0} \gamma(a, \varepsilon)=1, &
\end{array}
$$

$$
\begin{aligned}
& \lim _{\varepsilon \rightarrow 0} \sum_{a>0} a^{\alpha} e^{2 \pi i n \log a / \log \beta}\left[q_{a} \Omega_{n} \gamma(a, \varepsilon)+q_{-a} \bar{\Omega}_{-n} \gamma(-a, \varepsilon)\right] \\
= & \sum_{a>0} a^{\alpha} e^{2 \pi i n \log a / \log \beta}\left(q_{a} \Omega_{n}+q_{-a} \bar{\Omega}_{-n}\right) .
\end{aligned}
$$

This proves (2.9), except for the verification of our claim.

By direct substitution of (2.11) into (2.12), and a scaling of the integrand, we obtain

$$
g^{\varepsilon}(t)=\sum_{k=-\infty}^{\infty} \lambda^{k}\left(I_{1}(k)+I_{2}(k)+I_{3}(k)+I_{4}(k)\right)
$$

where

$$
\begin{aligned}
& I_{1}(k)=\frac{\beta^{k}}{\varepsilon} \int_{t}^{t+\varepsilon / \beta^{k}} q\left(\beta^{k} s\right) d s, \\
& I_{2}(k)=-\frac{\beta^{k}}{\varepsilon} \int_{0}^{\varepsilon / \beta^{k}} q\left(\beta^{k} s\right) d s, \\
& I_{3}(k)=-\beta^{k} \varepsilon \int_{t}^{t+1 / \beta^{k} \varepsilon} q\left(\beta^{k} s\right) d s, \\
& I_{4}(k)=\beta^{k} \varepsilon \int_{0}^{1 / \beta^{k} \varepsilon} q\left(\beta^{k} s\right) d s .
\end{aligned}
$$

Then, since $q(t)$ is bounded, say $|q(t)| \leq K_{1}$, we have for all $k \geq 0$,

If $k<0$, then we have for $\varepsilon \leq 1$,

$$
\left|I_{i}(k)\right| \leq K_{1} \quad \text { for all } i
$$

$$
\begin{aligned}
\left|I_{3}(k)+I_{4}(k)\right| & =\beta^{k} \varepsilon\left|\left[\int_{0}^{t}-\int_{1 /\left(\beta^{k} \varepsilon\right)}^{t+1 /\left(\beta^{k} \varepsilon\right)}\right] q\left(\beta^{k} s\right) d s\right| \\
& \leq 2 \beta^{k} \varepsilon K_{1} t \leq K_{2} \beta^{k}
\end{aligned}
$$


for $K_{2}=2 K_{1} \beta$. Also, if $k<0$, and $t \leq \varepsilon / \beta^{k}$,

$$
\begin{aligned}
\left|I_{1}(k)+I_{2}(k)\right| & =\frac{\beta^{k}}{\varepsilon}\left|\left[-\int_{0}^{t}+\int_{\varepsilon / \beta^{k}}^{t+\varepsilon / \beta^{k}}\right] q\left(\beta^{k} s\right) d s\right| \\
& \leq \frac{\beta^{k}}{\varepsilon} K_{3}\left[\frac{\beta^{k} t^{k}}{2}+\beta^{k} t\left(\frac{t}{2}+\varepsilon / \beta^{k}\right)\right] \leq 2 K_{3} t \beta^{k} \leq K_{4} \beta^{k}
\end{aligned}
$$

where $K_{4}=2 K_{3} \beta$, and $|q(t)| \leq K_{3} t$ on $[0,2]$. Here we use the facts that $t \leq \beta$ and $t \leq \varepsilon / \beta^{k}$.

Finally, if $k<0$ and $t \geq \varepsilon / \beta^{k}$ we have

$$
\begin{aligned}
\left|I_{1}(k)+I_{2}(k)\right| & =\frac{\beta^{k}}{\varepsilon}\left|\left[-\int_{0}^{\varepsilon / \beta^{k}}+\int_{t}^{t+\varepsilon / \beta^{k}}\right] q\left(\beta^{k} s\right) d s\right| \\
& \leq \frac{\beta^{k}}{\varepsilon} K_{3}\left[\frac{\beta^{k}}{2}\left(\frac{\varepsilon}{\beta^{k}}\right)^{2}+\beta^{k}\left(\frac{\varepsilon}{\beta^{k}}\right)\left(t+\frac{\varepsilon}{2 \beta^{k}}\right)\right] \\
& =K_{3}\left(\varepsilon+\beta^{k} t\right) \leq K_{4} \beta^{k},
\end{aligned}
$$

since $\varepsilon \leq \beta^{k} t$. We therefore have, for the $k^{\prime}$ th term in the series for $g^{\varepsilon}(t)$

$$
\left|\lambda^{k}\left(I_{1}(k)+I_{2}(k)+I_{3}(k)+I_{4}(k)\right)\right| \leq \begin{cases}K_{5} \lambda^{k} & \text { if } k \geq 0 \\ K_{5}(\lambda \beta)^{k} & \text { if } k<0,\end{cases}
$$

where $K_{5}=\max \left\{K_{1}, K_{2}+K_{4}\right\}$. Because the series $\sum_{k=-\infty}^{-1}(\lambda \beta)^{k}+\sum_{k=0}^{\infty} \lambda^{k}$ converges, we have a uniform bound on $\left|g^{\varepsilon}(t)\right|$, independent of $\varepsilon \leq 1$ and $t \in[1, \beta]$. Moreover, we may evaluate $\lim _{\varepsilon \rightarrow 0} g^{\varepsilon}(t)$ simply by taking a term by term limit:

$$
\begin{aligned}
& \lim _{\varepsilon \rightarrow 0} I_{1}(k)=q\left(\beta^{k} t\right) \\
& \lim _{\varepsilon \rightarrow 0} I_{2}(k)=-q(0)=0 \\
& \lim _{\varepsilon \rightarrow 0} I_{3}(k)=-\bar{q} \quad \text { where } \bar{q}=\lim _{T \rightarrow \infty} \frac{1}{T} \int_{0}^{T} q(t) d t \\
& \lim _{\varepsilon \rightarrow 0} I_{4}(k)=\bar{q},
\end{aligned}
$$

and so, for each $t$,

$$
\lim _{\varepsilon \rightarrow 0} g^{\varepsilon}(t)=\sum_{k=-\infty}^{\infty} \lambda^{k} q\left(\beta^{k} t\right)=g(t)
$$

Corollary. $g(t) \equiv 0$ implies $f(t)=-h(t)$ is smooth.

Since the fractal dimension of the graph of a smooth real valued function is one, we may summarize the results thus far as follows.

Theorem 2.4. Assume (H1) holds and $q$ is $C^{\prime}$. Suppose, further, that

$$
g_{\sigma}=0 \quad \text { for all } \sigma \text {. }
$$

Then $f(t)=-h(t)$ is smooth and $\operatorname{dim}(\operatorname{graph} f)=1$.

The nowhere differentiable case when some $g_{\sigma} \neq 0$. We next turn our attention to the case in which (2.14) does not hold, that is, $g_{\sigma_{0}} \neq 0$ for some $\sigma_{0}$. We will begin by 
demonstrating the existence of a constant $C_{1}>0$ such that for sufficiently small $L$,

$$
\max _{t \in J} f(t)-\min _{t \in J} f(t) \geq C_{1} L^{\alpha},
$$

where $J$ is any interval of length $L$. This in turn will imply that the number of $L$-balls necessary to cover the graph of $f$ on any unit interval is at least propositional to $L^{\alpha-1} \times L^{-1}=L^{\alpha-2}$. Hence

$$
\operatorname{dim}(\operatorname{graph} f) \geq \frac{\log k L^{\alpha-2}}{\log (1 / L)} \rightarrow 2-\alpha,
$$

as $L \rightarrow 0$, where $k$ is a constant of proportionality. The following proposition makes such estimates.

Proposition 2.5. Let

$$
I=\frac{\rho}{2 \pi n} \int^{2 \pi n / \rho} \gamma(t) \cos (\rho t+\phi) d t,
$$

where $\rho>0, \phi \in \mathbb{R}$, and $n>0$ is an integer. Then with $J=[0,2 \pi n / \rho]$,

$$
\sup _{J} \gamma-\inf _{J} \gamma \geq \pi|I| \text {. }
$$

Proof. Without loss of generality we can add a constant to $\gamma$, as that does not affect the value of $I$. If the result is false then upon adding an appropriate constant we have, for some $\varepsilon$,

$$
|\gamma(t)| \leq \frac{\pi}{2}|I|-\varepsilon, \quad \text { for all } t \in J
$$

This implies

$$
|I| \leq \frac{\rho}{2 \pi n} \int_{0}^{2 \pi n / \rho}\left(\frac{\pi}{2}|I|-\varepsilon\right)|\cos (\rho t+\phi)| d t=|I|-\frac{2 \varepsilon}{\pi},
$$

which is a contradiction.

Now to estimate the integral of the above proposition, we require several technical lemmas.

LEMMA 2.6. Let $p: \mathbb{R} \rightarrow \mathbb{R}$ be almost periodic with Fourier series $p(t) \sim \sum_{\mu} p_{\mu} e^{i \mu t}$. Suppose $p_{\rho}=0$ for some $\rho>0$. Then for any $\phi \in \mathbb{R}$ and any integer $n>0$,

$$
\frac{\rho}{2 \pi n}\left|\int_{0}^{2 \pi n / \rho} p(t) \cos (\rho t+\phi) d t\right| \leq 2 \sum_{\mu>0}\left|p_{\mu}\right| \frac{\min \{1 / \pi n, \mu / \rho,|(\mu / \rho)-1|\}}{|(\mu / \rho)-1|}
$$

Proof. Consider first the case $p(t)=p_{\mu} e^{i \mu t}+p_{-\mu} e^{-i \mu t}$, so that $p(t)=2\left|p_{\mu}\right| \cos (\mu t+\theta)$ for some $\theta \in \mathbb{R}$. Thus we estimate

$$
\begin{aligned}
\int_{0}^{2 \pi n / \rho} & \cos (\mu t+\theta) \cos (\rho t+\phi) d t \\
= & \frac{\sin (\mu t+\rho t+\theta+\phi)}{2(\mu+\rho)}+\left.\frac{\sin (\mu t-\rho t+\theta-\phi)}{2(\mu-\rho)}\right|_{0} ^{2 \pi n / \rho} \\
= & \frac{\sin ((2 \pi n \mu / \rho)+\theta+\phi)-\sin (\theta+\phi)}{2(\mu+\rho)}+\frac{\sin ((2 \pi n \mu / \rho)+\theta-\phi)-\sin (\theta-\phi)}{2(\mu-\rho)} .
\end{aligned}
$$


The absolute value of this quantity is at most $\min \{2,2 \pi n \mu / \rho, 2 \pi n|(\mu / \rho)-1|\} /|\mu-\rho|$ which gives the required estimate.

It follows next that if $\sum\left|p_{\mu}\right|<\infty$, the lemma is easily proved by integrating term by term.

Consider the general case. Without loss we assume

$$
\sum_{\mu<1}\left|p_{\mu} \mu\right|+\sum_{\mu \geqq 1}\left|p_{\mu} \mu^{-1}\right|<\infty
$$

if this is not so then the right-hand side of $(2.16)$ is infinite and the result is trivially true. Define

$$
p_{\varepsilon}(t)=\frac{1}{\varepsilon} \int_{t}^{t+\varepsilon} p(s) d s-\varepsilon \int_{t}^{t+1 / \varepsilon} p(s) d s .
$$

Note that $p_{\varepsilon}$ is almost periodic and, as $\varepsilon \rightarrow 0$, converges uniformly on $\mathbb{R}$ to $p(t)-\bar{p}$ where $\bar{p}$ is the mean value of $p$. Moreover, with $\gamma(\mu, \varepsilon)$ as before,

For any fixed $\varepsilon>0$,

$$
p_{\varepsilon}(t) \sim \sum_{\mu} p_{\mu} \gamma(\mu, \varepsilon) e^{i \mu t} .
$$

$$
\sum_{\mu}\left|p_{\mu} \gamma(\mu, \varepsilon)\right| \leq K(\varepsilon)\left(\sum_{\mu<1}\left|p_{\mu} \mu\right|+\sum_{\mu \geq 1}\left|p_{\mu} \mu^{-1}\right|\right)<\infty,
$$

for some constant $K(\varepsilon)$. Hence the lemma holds with $p_{\varepsilon}(t)$ replacing $p(t)$ and $p_{\mu} \gamma(\mu, \varepsilon)$ replacing $p_{\mu}$. We may take the limit as $\varepsilon \rightarrow 0$ because of the uniform convergence of $p_{\varepsilon}(t)$, and because $\gamma(\mu, \varepsilon) \rightarrow 1$ as $\varepsilon \rightarrow 0$,

$$
\sup _{\substack{\mu>0 \\ \varepsilon>0}}|\gamma(\mu, \varepsilon)|<\infty \text {. }
$$

We next apply lemma 2.6 to any translate of the function

$$
\begin{aligned}
f_{k}(t) & =f(t)-\left(\sum_{m=0}^{\infty} \lambda^{m} q_{\sigma_{0} \beta^{k-m}}\right) e^{i \sigma_{0} \beta^{k_{t}}}-\left(\sum_{m=0}^{\infty} \lambda^{m} q_{-\sigma_{0} \beta^{k-m}}\right) e^{-i \sigma_{0} \beta^{k_{t}}} \\
& =f(t)-2\left|\sum_{m=0}^{\infty} \lambda^{m} q_{\sigma_{0} \beta^{k-m}}\right| \cos \left(\sigma_{0} \beta^{k} t+\phi_{k}\right)
\end{aligned}
$$

for some $\phi_{k} \in \mathbb{R}$. Note that $f_{k}$ is simply the function $f$ with the terms $e^{ \pm i \sigma_{o} \beta^{k}}$ removed from its Fourier series. Setting $\rho=\sigma_{0} \beta^{k}$ and choosing any $\theta \in \mathbb{R}$, we obtain with $\phi=\sigma_{0} \beta^{k} \theta+\phi_{k}$,

$$
\begin{aligned}
& \left|\frac{\sigma_{0} \beta^{k}}{2 \pi n} \int_{0}^{2 \pi n /\left(\sigma_{0} \beta^{k}\right)} f(t+\theta) \cos \left(\sigma_{0} \beta^{k} t+\phi\right) d t-\right| \sum_{m=0}^{\infty} \lambda^{m} q_{\sigma_{0} \beta^{k-m}}|| \\
& \quad=\left|\frac{\sigma_{0} \beta^{k}}{2 \pi n} \int_{0}^{2 \pi n /\left(\sigma_{0} \beta^{k}\right)} f_{k}(t+\theta) \cos \left(\sigma_{0} \beta^{k} t+\phi\right) d t\right| \\
& \quad \leq 2 \sum_{a>0} \sum_{m=0}^{\infty} \lambda^{m}\left|q_{a}\right| \frac{\min \left\{1 / \pi n, a \beta^{m} /\left(\sigma_{0} \beta^{k}\right),\left|\left(a \beta^{m} / \sigma_{0} \beta^{k}\right)-1\right|\right\}}{\left|\left(a \beta^{m} / \sigma_{0} \beta^{k}\right)-1\right|} \\
& \quad \leq 2 \lambda^{k} \sum_{a>0} \sum_{r=-\infty}^{\infty * *} \lambda^{r}\left|q_{a}\right| \frac{\min \left\{\sigma_{0} / \pi n, a \beta^{r},\left|a \beta^{r}-\sigma_{0}\right|\right\}}{\left|a \beta^{r}-\sigma_{0}\right|} \\
& \quad \operatorname{def}^{-}=2 \lambda^{k} \Phi(\varepsilon), \quad \text { where } \varepsilon=\sigma_{0} / \pi n .
\end{aligned}
$$


In the double summations above, ${ }^{*}$ denotes the omission of terms $(a, m)$ with $a \beta^{m}=\sigma_{0} \beta^{k}$, and ${ }^{* *}$ the omission of terms for which $a \beta^{r}=\sigma_{0}$. From (2.17) we have

$$
\begin{aligned}
\frac{\sigma_{0} \beta^{k}}{2 \pi n} & \int_{0}^{2 \pi n /\left(\sigma_{0} \beta^{k}\right)} f(t+\theta) \cos \left(\sigma_{0} \beta^{k} t+\phi\right) d t \\
& \left.\geq\left|\sum_{m=0}^{\infty} \lambda^{m} q_{\sigma_{0} \beta^{k-m}}\right|-2 \lambda^{k} \Phi\left(\sigma_{0} / \pi n\right) \quad \text { (using }|A-B|=C \Rightarrow A \geq-|B|-C\right) \\
& =\lambda^{k}\left[\left|\sum_{r=-k}^{\infty} \lambda^{r} q_{\sigma_{0} \beta^{-r}}\right|-2 \Phi\left(\sigma_{0} / \pi n\right)\right] \\
& =\lambda^{k}\left[\left|g_{\sigma_{0}}\right|+o(1)-2 \Phi\left(\sigma_{0} / \pi n\right)\right] \quad \text { where } o(1) \rightarrow 0 \text { as } k \rightarrow \infty .
\end{aligned}
$$

A final estimate choosing $n$ so that $\Phi\left(\sigma_{0} / \pi n\right)$ is small; because $g \sigma_{0} \neq 0$, this will give a lower bound of order $O\left(\lambda^{k}\right)$ for the integral of (2.18), for some $n$ independent of $k$.

LEMMA 2.7. $\Phi(\varepsilon) \rightarrow 0$ as $\varepsilon \rightarrow 0$.

Proof. We have

$$
\Phi(\varepsilon)=\sum_{a>0} \sum_{r=-\infty}^{\infty *} \lambda^{r}\left|q_{a}\right| \frac{\min \left\{\varepsilon, a \beta^{r},\left|a \beta^{r}-\sigma_{0}\right|\right\}}{\left|a \beta^{r}-\sigma_{0}\right|} .
$$

Each term in this series tends monotonically to zero as $\varepsilon \rightarrow 0$. Therefore it is sufficient to show $\Phi(\varepsilon)<\infty$ for some $\varepsilon$ in order to conclude $\Phi(\varepsilon) \rightarrow 0$. Fix $\varepsilon<\sigma_{0}$ and consider three ranges of $r$ :

$$
\begin{aligned}
& r \leq r_{0} \quad \text { where } a \beta^{r}<\varepsilon, \\
& r_{0}<r \leq r_{1} \quad \text { where } \varepsilon \leq a \beta^{r}<2 \sigma_{0}, \\
& r_{1}<r \quad \text { where } a \beta^{r} \geq 2 \sigma_{0} .
\end{aligned}
$$

In the first range $a \beta^{r} /\left|a \beta^{r}-\sigma_{0}\right| \leq a \beta^{r} /\left(\sigma_{0}-\varepsilon\right)=K_{1} a \beta^{r}$; in the third range $\varepsilon /\left|a \beta^{r}-\sigma_{0}\right| \leq K_{2}\left(a \beta^{r}\right)^{-1}$ for some $K_{2}$. In the second range use the quantity $\left|a \beta^{r}-\sigma_{0}\right|$ in the minimum in the formula for $\Phi(\varepsilon)$. We now have

$$
\begin{aligned}
& \sum_{r=-\infty}^{\infty * *} \lambda^{r} \frac{\min \left\{\varepsilon, a \beta^{r},\left|a \beta^{r}-\sigma_{0}\right|\right\}}{\left|a \beta^{r}-\sigma_{0}\right|} \\
& \quad \leq \sum_{r=-\infty}^{r_{0}} K_{1} a(\lambda \beta)^{r}+\sum_{r=r_{0}+1}^{r_{1}} \lambda^{r}+\sum_{r=r_{1}+1}^{\infty}\left(K_{2} / a\right)(\lambda / \beta)^{r} .
\end{aligned}
$$

Summing each of these geometric series, using the definitions of $r_{0}$ and $r_{1}$, and in particular that $\lambda=\beta^{-\alpha}$, we obtain an upper bound $K_{3} a^{\alpha}$ for $(2.19)$, for some $K_{3}$. Therefore

$$
\Phi(\varepsilon) \leq \sum_{a>0} K_{3} a^{\alpha}\left|q_{a}\right|<\infty
$$

by (H1).

We may now use the above results to obtain the lower bound (2.15) for the variation of $f$ over small intervals. 
Proposition 2.8. Let (H1) hold and let $\mathrm{g}_{\sigma_{0}} \neq 0$. Then there exist $C_{1}>0$ and $L_{0}>0$ such that

$$
\max _{J} f-\min _{J} f \geq C_{1} L^{\alpha}
$$

whenever length $(J)=L \leq L_{0}$.

Proof. Choose $K_{1}>0$ and a sufficiently large integer $n$ so that $0<K_{1}<$ $\pi\left|g_{\sigma_{0}}\right|-2 \Phi\left(\sigma_{0} / \pi n\right)$; this can be done by lemma 2.7. Set $K_{2}=2 \pi n / \sigma_{0}$. Applying proposition 2.5 to $(2.18)$ shows that for large $k$,

$$
\max _{J} f-\min _{J} f \geq K_{1} \lambda^{k}=K_{1} \beta^{-\alpha k}
$$

whenever length $(J) \geq K_{2} \beta^{-k}$. For a given $L$, choosing $k$ so that $K_{2} \beta^{-k} \leq L \leq$ $K_{2} \beta^{-k+1}$ easily gives the result.

The lower bound on $\max _{J} f-\min _{J} f$ of the above proposition will be used to obtain a lower bound for the fractal dimension, as well as for the dimension of the attractor of the dynamical system (1.4). Obtaining similar upper bounds gives the exact value of the dimension. Fortunately, such upper bounds are much more easily obtained than lower bounds.

Proposition 2.9. Let $q: \mathbb{R} \rightarrow \mathbb{R}$ be $C^{\prime}$, and suppose both $q(t)$ and $q^{\prime}(t)$ are bounded for $t \in \mathbb{R}$. Then there exists $C_{2}>0$ such that

$$
\max _{J} f-\min _{J} f \leq C_{2} L^{\alpha}
$$

whenever length $(J)=L$.

Proof. We show $\left|f\left(t_{1}\right)-f\left(t_{2}\right)\right| \leq C_{2}\left|t_{1}-t_{2}\right|^{\alpha}$. Assuming $|q(t)|,\left|q^{\prime}(t)\right| \leq K_{3}$ for all $t$, let $\beta^{-n_{0}-1} \leq\left|t_{1}-t_{2}\right| \leq \beta^{-n_{0}}$. Therefore

$$
\begin{aligned}
\left|q\left(\beta^{n} t_{1}\right)-q\left(\beta^{n} t_{2}\right)\right| & \leq \min \left\{2 K_{3}, K_{3} \beta^{n}\left|t_{1}-t_{2}\right|\right\} \\
& \leq \begin{cases}2 K_{3} & n>n_{0} \\
K_{3} \beta^{n-n_{0}} & n \leq n_{0},\end{cases}
\end{aligned}
$$

so estimating term by term in the series (1.1) gives

$$
\begin{aligned}
\left|f\left(t_{1}\right)-f\left(t_{2}\right)\right| & \leq 2 K_{3} \sum_{n=n_{0}+1}^{\infty} \lambda^{n}+K_{3} \sum_{n=-\infty}^{n_{0}}(\lambda \beta)^{n} \beta^{-n_{0}} \\
& \leq K_{4} \lambda^{n_{0}}=K_{4} \beta^{-\alpha n_{0}} \leq C_{2}\left|t_{1}-t_{2}\right|^{\alpha} .
\end{aligned}
$$

For a small interval $J$ of length $L$, how many squares of size $L \times L$ are needed to cover the graph of $f$ where $f$ is restricted to $J$, assuming (H1) and (H2)? By (2.20) we need at least $C_{1} L^{\alpha-1}$ and at most $C_{2} L^{\alpha-1}+1$ by (2.21). The number of intervals of length $L$ in $[0,1]$ is approximately $1 / L$, so to cover the graph of $f$ on $[0,1]$ the number of squares $N(L)$ needed to cover the graph is proportional to $L^{-1} \times L^{\alpha-1}$. Actually we may not have chosen our boxes optimally, but optimal positioning decreases the number of boxes negligibly, by at most a factor of 2 . Hence

$$
\frac{\log N(L)}{\log 1 / L} \rightarrow 2-\alpha \quad \text { as } L \rightarrow 0
$$


Summarizing we have the following result.

THEOREM 2.10. Assume (H1) and $g_{\sigma_{0}} \neq 0$. Assume also that $q^{\prime}(t)$ is uniformly bounded on $(-\infty, \infty)$. Then

$$
\operatorname{dim}(\operatorname{graph} f)=2-\alpha=2-|\log \lambda / \log \beta| .
$$

Since the convergence condition $(\mathrm{H} 1)$ is satisfied by trigonometric polynomials, theorem $\mathbf{A}$ is proved.

\section{The dimension of an attracting torus}

We turn our attention here to the dynamical system $(1.4),(1.5)$; recall the formula

$$
F(x, y)=(A x, \lambda y+p(x))
$$

for $F: T^{k} \times \mathbb{R} \rightarrow T^{k} \times \mathbb{R}$, and the explicit formula for the attractor is

where

$$
y=\phi\left(A^{-1} x\right)
$$

$$
\phi(x)=\sum_{n=0}^{\infty} \lambda^{n} p\left(A^{-n} x\right),
$$

where $A$ is a $k \times k$ matrix as described in the introduction. In this section we apply our previous results to prove theorem $B$, the $2 \times 2$ case with $A=A_{*}$, that is, to calculate $\operatorname{dim}($ graph $\phi$ ). We do this by first restricting $\phi$ to a dense one-dimensional submanifold $M$ of $T^{k}$; this restriction gives a function $f$ of the type (1.1) considered in $\S 2$. Results of that section (theorem 2.4, propositions 2.8 and 2.9) and additional results on the smoothness of $\phi$ in a direction transverse to $M$ (proposition 3.4) are used to prove theorem $B$. Throughout this section we assume the hypotheses of theorem $\mathrm{B}$, namely that $p: T^{2} \rightarrow \mathbb{R}$ is $C^{3}$ and $\lambda \in\left(B_{*}, 1\right)$, where $B_{*}$ is the minimum of the absolute values of the eigenvalues of $A$. We let $w_{*}$ denote the corresponding eigenvector. As before, we assume without loss that $p(0)=0$. In general $p: T^{k} \rightarrow \mathbb{R}$ is $C^{k}$. We leave to the reader the very minor modifications needed for theorem $C$.

The manifold $M$ is defined to be the line through 0 in the direction $w_{*}$. Therefore $M=\{x=(u, v) \bmod 1: v=\mu u\}$, where $\mu=-(1+\sqrt{5}) / 2$. In particular $w_{*}=(1, \mu)$ is an eigenvector of $A_{*}$. Observe $M$ is dense in $T^{2}$, as $\mu$ is irrational. To restrict $\phi$ to $M$, parameterize $M$ by setting $u=t, v=\mu t$, $\bmod 1$ for $t \in \mathbb{R}$; then using the fact that $B^{-1}$ is an eigenvalue, specifically

we obtain

$$
A^{-1}\left(\begin{array}{c}
1 \\
\mu
\end{array}\right)=B\left(\begin{array}{l}
1 \\
\mu
\end{array}\right),
$$

$$
\begin{aligned}
f(t) \stackrel{\text { def }}{=} \phi(t, \mu t) & =\sum_{n=0}^{\infty} \lambda^{n} p\left(B^{n} t, B^{n} \mu t\right) \\
& =\sum_{n=0}^{\infty} \lambda^{n} q\left(B^{n} t\right),
\end{aligned}
$$

where $q(t) \stackrel{\text { def }}{=} p(t, \mu t)$. The function $q$ is almost periodic. With enough smoothness on $p$, the various hypotheses of $\S 2$ hold for $q$. When $k>2$ we need $p$ to be $C^{k+1}$ for $(\mathrm{H} 1)$ to hold. 
LeMMA 3.1. The function $q$ is $C^{3}$, almost periodic, and satisfies ( $\left.\mathrm{H} 1\right)$, and $q^{\prime}(t)$ is bounded for all $t$.

Proof. We only verify the statement about (H1) as the other claims are easily proved. Consider first the Fourier series for $p$ :

$$
p(u, v) \sim \sum_{n=-\infty}^{\infty} \sum_{m=-\infty}^{\infty} p_{m n} e^{2 \pi i(m u+n v)}
$$

with coefficients $p_{m n}$. Because $p$ is at least $C^{3}$, the functions $\partial^{3} p / \partial u^{3}$ and $\partial^{3} p / \partial v^{3}$ have Fourier series with corresponding coefficients $(2 \pi i m)^{3} p_{m n}$ and $(2 \pi i n)^{3} p_{m n}$. Moreover, these coefficients are bounded in $m$ and $n$. Thus for some $K_{1}>0$

$$
\left|p_{m n}\right| \leq \frac{K_{1}}{1+|m|^{3}+|n|^{3}} .
$$

It follows that the series (3.1) is absolutely convergent, and also that

$$
\begin{aligned}
q(t) & =\sum_{n=-\infty}^{\infty} \sum_{m=-\infty}^{\infty} p_{m n} e^{2 \pi i(m+n \mu) t} \\
& =\sum_{a} q_{a} e^{i a t}
\end{aligned}
$$

where $q_{2 \pi(m+n \mu)}=p_{m n}$. Thus

$$
\begin{aligned}
\sum_{a}\left|q_{a}\right||a|^{\alpha} & =(2 \pi)^{\alpha} \sum_{n=-\infty}^{\infty} \sum_{m=-\infty}^{\infty}\left|p_{m n}\right||m+n \mu|^{\alpha} \\
& \leq(2 \pi)^{\alpha} \sum_{n=-\infty}^{\infty} \sum_{m=-\infty}^{\infty} \frac{K_{1}|\mu|^{\alpha}(|m|+|n|)^{\alpha}}{1+|m|^{3}+|n|^{3}} \\
& \leq K_{2} \sum_{n=-\infty}^{\infty} \sum_{m=-\infty}^{\infty} \frac{1}{(1+|m|+|n|)^{3-\alpha}}<\infty
\end{aligned}
$$

since $3-\alpha>2$. (Recall $0<\alpha<1$.) This proves (H1) holds.

For general $k$, the $p$ coefficients have $k$ subscripts and $k$ nested sums are needed so we need the function $p$ to be $C^{k+1}$ for $(H 1)$ to be satisfied.

We will calculate $\operatorname{dim}(\operatorname{graph} \phi)$ by estimating $\max _{E} \phi-\min _{E} \phi$ where $E$ is a small neighbourhood of an arbitrary point $\left(u_{0}, v_{0}\right) \in T^{2}$. To this end we first introduce a special coordinate system $(t, s)$ about the point $\left(u_{0}, v_{0}\right)$. Let

$$
\Phi\left(t, s ; u_{0}, v_{0}\right)=\phi\left(u_{0}+t-\mu s, v_{0}+\mu t+s\right) .
$$

Observe that the $t$-direction is parallel to the manifold $M$, and the $s$-direction is perpendicular in this coordinate system. Indeed, $f(t)=\Phi(t, 0 ; 0,0)$. Using the relations

we see that

$$
A^{-1}\left(\begin{array}{c}
1 \\
\mu
\end{array}\right)=B\left(\begin{array}{c}
1 \\
\mu
\end{array}\right) \text { and } A^{-1}\left(\begin{array}{c}
-\mu \\
1
\end{array}\right)=\frac{1}{B}\left(\begin{array}{c}
-\mu \\
1
\end{array}\right)
$$

$$
\begin{aligned}
\Phi\left(t, s ; u_{0}, v_{0}\right) & =\sum_{n=0} \lambda^{n} p\left[A^{-n}\left(\begin{array}{l}
u_{0} \\
v_{0}
\end{array}\right)+A^{-n}\left(\begin{array}{c}
1 \\
\mu
\end{array}\right) t+A^{-n}\left(\begin{array}{c}
-\mu \\
1
\end{array}\right) s\right] \\
& =\sum_{n=0}^{\infty} \lambda^{n} p\left[A^{-n}\left(\begin{array}{c}
u_{0} \\
v_{0}
\end{array}\right)+B^{n}\left(\begin{array}{c}
1 \\
\mu
\end{array}\right) t+B^{-n}\left(\begin{array}{c}
-\mu \\
1
\end{array}\right) s\right] .
\end{aligned}
$$


Proposition 3.4. $\Phi$ is a $C^{1}$ function of $s$, and $|\partial \Phi / \partial s|$ is bounded independent of $t, s, u_{0}$, and $v_{0}$. In fact

$$
\frac{\partial \Phi}{\partial s}\left(t, s ; u_{0}, v_{0}\right)=\left.\sum_{n=0}^{\infty}\left(\lambda B^{-1}\right)^{n}\left(-\mu \frac{\partial p}{\partial u}+\frac{\partial p}{\partial v}\right)\right|_{\chi_{n}},
$$

with

$$
\chi_{n}=A^{-n}\left(\begin{array}{c}
u_{0} \\
v_{0}
\end{array}\right)+B^{n}\left(\begin{array}{c}
1 \\
\mu
\end{array}\right) t+B^{-n}\left(\begin{array}{c}
-\mu \\
1
\end{array}\right) s
$$

The proof is similar to that of lemma 2.1. For theorem $\mathrm{C}$ one needs to examine the variation of $\phi$ in all eigenvector directions other than $w_{*}$ and one concludes in case (i) that the variation of $\phi$ on an $\varepsilon$-cube (i.e. $\max \phi-\min \phi$ ) is due primarily to the variation in the $w_{*}$ direction.

The next lemma is the final link needed for theorem B, case (ii) (i.e. the case of smooth $\phi$ ).

LEMMA 3.5. Let each Fourier coefficient $g_{\sigma}=0$, where $g(t)=\sum_{n=-\infty}^{\infty} \lambda^{n} q\left(B^{n} t\right)$. Then $\Phi$ is a $C^{1}$ function of $(t, s)$.

Proof. By proposition 2.2, $g \equiv 0$ so

$$
\begin{aligned}
\phi(t, \mu t) & =f(t)=-\sum_{n=-\infty}^{-1} \lambda^{n} q\left(B^{n} t\right) \\
& =-\sum_{n=-\infty}^{-1} \lambda^{n} p\left(A^{-n}\left(\begin{array}{l}
1 \\
\mu
\end{array}\right) t\right) .
\end{aligned}
$$

By theorem 2.4, this function is $C^{1}$, but we need a little more. Because $M=\{(t, \mu t): t \in \mathbb{R}\}$ is dense in $T^{2}$, we may take a limit $\left(t_{k}, \mu t_{k}\right) \rightarrow(u, v)$ of a sequence of points on $M$ in the above expression, to obtain for any $(u, v) \in T^{2}$

$$
\phi(u, v)=-\sum_{n=-\infty}^{-1} \lambda^{n} p\left(A^{-n}\left(\begin{array}{l}
u \\
v
\end{array}\right)\right)
$$

Hence

$$
\begin{aligned}
\Phi\left(t, s ; u_{0}, v_{0}\right) & =-\sum_{n=-\infty}^{-1} \lambda^{n} p\left(A^{-n}\left(\begin{array}{l}
u_{0} \\
v_{0}
\end{array}\right)+A^{-n}\left(\begin{array}{c}
1 \\
\mu
\end{array}\right) t+A^{-n}\left(\begin{array}{c}
-\mu \\
1
\end{array}\right) s\right) \\
& =-\sum_{n=-\infty}^{-1} \lambda^{n} p\left(A^{-n}\left(\begin{array}{l}
u_{0} \\
v_{0}
\end{array}\right)+B^{n}\left(\begin{array}{c}
1 \\
\mu
\end{array}\right) t+B^{-n}\left(\begin{array}{c}
-\mu \\
1
\end{array}\right) s\right) .
\end{aligned}
$$

Let $\chi_{n}$ be the argument of $p$ above. The proof is completed by showing

$$
\frac{\partial \Phi}{\partial t}\left(t, s ; u_{0}, v_{0}\right)=-\left.\sum_{n=-\infty}^{-1}(\lambda B)^{n}\left(\frac{\partial p}{\partial u}+\mu \frac{\partial p}{\partial v}\right)\right|_{\chi_{n}}
$$

again by the methods of lemma 2.1 . Therefore $\partial \Phi / \partial t$ exists and is continuous. This together with proposition 3.4 implies $\Phi$ is a $C^{1}$ function of $(t, s)$, for any $\left(u_{0}, v_{0}\right)$.

Notice theorem $C$ does not claim $\phi$ is $C^{1}$ in case (ii) so the lines $w_{i} t$ are not assumed to be dense. 
Proof of theorem B. If $g_{\sigma}=0$ for each $\sigma$, then conclusion (ii) holds; for by proposition 3.4, $\phi: T^{2} \rightarrow \mathbb{R}$ is a $C^{1}$ function, so its graph is two dimensional.

Suppose therefore $g_{\sigma_{0}} \neq 0$ for some $\sigma_{0}$. We shall obtain conclusion (i), namely that

$$
\operatorname{dim}(\operatorname{graph} \phi)=3-\left|\frac{\log \lambda}{\log B}\right| \text {. }
$$

Fix $\left(u_{0}, v_{0}\right) \in T^{2}$ and let $E_{L}$ be the box $|t| \leq \frac{1}{2} L,|s| \leq \frac{1}{2} L$ centred at $\left(u_{0}, v_{0}\right)$. The manifold $M$ is given by the line $\left(u_{0}+t-\mu s_{0}, v_{0}+\mu t+s_{0}\right)$ as a function of $t$ for a dense set of $s_{0}$; fix one such $s_{0}$ with $\left|s_{0}\right| \leq \frac{1}{2} L$. Then $t \rightarrow \Phi\left(t, s_{0} ; u_{0}, v_{0}\right)$ is simply a translate of $f(t)$, so by propositions 2.8 and 2.9

$$
C_{1} L^{\alpha} \leq \max _{|t| \leq \frac{1}{2} L} \Phi\left(t, s_{0} ; u_{0}, v_{0}\right)-\min _{|t| \leq \frac{1}{2} L} \Phi\left(t, s_{0} ; u_{0}, v_{0}\right) \leq C_{2} L^{\alpha},
$$

provided $L \leq L_{0}$. Also, by proposition 3.4 with the Lipschitz constant

$$
\sup _{\left(t, s, u_{0}, v_{0}\right)}\left|\frac{\partial \Phi}{\partial s}\right|=K_{11}
$$

in $s$, we have

$$
\begin{aligned}
C_{1} L^{\alpha}-2 K_{11} L & \leq \max _{E_{L}} \Phi\left(t, s ; u_{0}, v_{0}\right)-\min _{E_{L}} \Phi\left(t, s ; u_{0}, v_{0}\right) \\
& \leq C_{2} L^{\alpha}+2 K_{11} L .
\end{aligned}
$$

If $L$ is sufficiently small, in fact if $L^{1-\alpha} \leq \min \left\{C_{2} / 2 K_{11}, C_{1} / 4 K_{11}\right\}$, we have

$$
\frac{1}{2} C_{1} L^{\alpha} \leq \max _{E_{L}} \Phi\left(t, s ; u_{0}, v_{0}\right)-\min _{E_{L}} \Phi\left(t, s ; u_{0}, v_{0}\right) \leq 2 C_{2} L^{\alpha} .
$$

These bounds hold uniformly for $\left(u_{0}, v_{0}\right) \in T^{2}$ and small $L$. The number of $L$ balls needed to cover the graph of $\phi$ is therefore proportional to $L^{\alpha-1} \times 1 / L^{2}=L^{\alpha-3}$. Hence,

$$
\begin{aligned}
\operatorname{dim}(\operatorname{graph} \phi) & =3-\alpha \\
& =3-\left|\frac{\log \lambda}{\log B}\right|
\end{aligned}
$$

Alterations for theorem $\mathrm{C}$. In the general case we choose the eigenvectors of $\boldsymbol{A}$ to be coordinate axes in $T^{k}$ and we must estimate the variation (i.e. $\max \phi-\min \phi$ ) on $\varepsilon$-cubes in $T^{k}$.

In case (i), this variation is essentially due to the variation along $M$, that is the variation in the direction $w_{*}$. As argued above the number of $L$ balls needed to cover the graph is proportional to $L^{\alpha-1} \times 1 / L^{k}=L^{\alpha-k-1}$. Hence

$$
\operatorname{dim}(\operatorname{graph} \phi)=k+1-\left|\frac{\log \lambda}{\log B_{*}}\right|
$$

On the other hand $\prod^{k}\left|B_{i}\right|=1$ and $1>\lambda>B_{*}$ so that the product of the $k$ largest Lyapunov numbers is $\left|B_{1}\right| \times \cdots \times\left|B_{k-1}\right| \times \lambda>1$, which is $\lambda / B_{*}$. The integer part $m$ of the Lyapunov dimension is $k$ and so

$$
\operatorname{dim}_{\text {Lyap }}=k+\left|\frac{\log \left(\lambda / B_{*}\right)}{\log B_{*}}\right|=k+1-\left|\frac{\log \lambda}{\log B_{*}}\right| .
$$


This research was partially supported under NSF Grants MCS 78-18221, MCS 8117967 and MCS 82-0718, and Air Force Office of Scientific Research Grant 81-0217A, and Department of Energy Grant DE-ASO5-82ER12026.

\section{REFERENCES}

[1] J. C. Alexander \& J. A. Yorke. Fat baker's transformations. Ergod. Th. \& Dynam. Sys. 4, (1984), 1-24.

[2] M. V. Berry \& Z. V. Lewis. Proc. Royal Soc. London A370 (1980), 459-484.

[3] A. S. Besicovitch \& H. D. Ursell. J. London Math. Soc. 12 (1937) 18-25.

[4] C. Corduneanu. Almost Periodic Functions. Interscience: New York, 1968.

[5] K. J. Falconer. The Geometry of Fractal Sets. Cambridge Univ. Press, in press.

[6] J. D. Farmer, E. Ott \& J. A. Yorke. The dimension of chaotic attractors. Phys. D. To appear.

[7] P. Frederickson, J. L. Kaplan, E. D. Yorke \& J. A. Yorke. The Lyapunov dimension of strange attractors. J. Differential Equations. To appear.

[8] G. H. Hardy. Weierstrass's non-differentiable function. Trans. Amer. Math. Soc. 17 (1916), 301-325.

[9] J. L. Kaplan \& J. A. Yorke. Chaotic behavior of multidimensional difference equations. In Functional Differential Equations and Approximation of Fixed Points (H. O. Peitgen and H. O. Walther, eds.). Springer Verlag Lecture Notes in Math \#730 (1979), 228-237.

[10] S. A. Kline. J. London Math. Soc. 20 (1945) 79-86.

[11] F. Ledrappier. Some relations between dimension and Lyapunov exponents. Commun. Math. Phys. 81 (1981), 229-238.

[12] E. R. Love \& L. C. Young. Fundamental Math. 28 (1937) 243-257.

[13] J. Moser. On a theorem of Anosov. J. Differential Equations 5 (1969), 411-490. 